\title{
Tyrosine Kinase-Dependent Defense Responses Against Herbivory in Arabidopsis
}

\section{Takumi Miyamoto', Takuya Uemura', Keiichirou Nemoto², Maho Daito ${ }^{1}$, Akira Nozawa ${ }^{3}$, Tatsuya Sawasaki ${ }^{3}$ and Gen-ichiro Arimura ${ }^{1 *}$}

${ }^{1}$ Department of Biological Science and Technology, Faculty of Industrial Science and Technology, Tokyo University of Science, Tokyo, Japan, ${ }^{2}$ Iwate Biotechnology Research Center, Iwate, Japan, ${ }^{3}$ Proteo-Science Center, Ehime University, Matsuyama, Japan

Tyrosine (Tyr) phosphorylation (TP) is important for promotion of plants' signaling. Arabidopsis calcium-dependent protein kinase related protein kinases (CRK2 and CRK3) phosphorylate Tyr residues of a subset of transcription factors (TFs), including herbivory-responsive ethylene response factor 13 (ERF13), but the in vivo functions of these kinases in plant defense responses and development remain to be clarified. We show that when CRKs were coexpressed with ERF13 in Arabidopsis leaf protoplasts, the transcription activity regulated via ERF13 was elevated by CRK2 but not CRK3 or their kinase-dead form mutants. Moreover, this elevation was abolished when a Tyr-phosphorylation mutant of ERF was coexpressed with CRK2, indicating that CRK2 serves as an effector of ERF13 mediated by Tyr-phosphorylation. Moreover, CRK2 and CRK3 acted as effectors of RAP2.6 and WRKY14, respectively. CRK-overexpressing lines and knockout mutants of Arabidopsis plants showed increased and decreased expression levels of the defensin gene PDF1.2 in leaves, respectively, conferring on the plants modulated defense properties against the generalist herbivore Spodoptera litura. However, these lines did not show any obvious developmental defects, indicating that CRKs play a role in defense responses but not in the ordinary growth or development of plants. Transcription of both CRK2 and CRK3 was positively regulated by jasmonate signaling and abscisic acid (ABA) signaling upon herbivory. Our findings suggest that these phytohormone-responsive CRKs work coordinately for plant defense responses via Tyr phosphorylation of herbivory-responsive regulators.

\footnotetext{
Keywords: Arabidopsis thaliana, calcium-dependent protein kinase related protein kinase (CRK), defense response, Spodoptera litura, tyrosine kinase
}

\section{INTRODUCTION}

Tyrosine (Tyr) phosphorylation (TP) is a notable regulator of signal transduction in eukaryotic cells (Blume-Jensen and Hunter, 2001). It has been estimated that in Arabidopsis thaliana (Arabidopsis) and rice, $4 \%$ of phosphopeptides are Tyr-phosphorylated peptides, which is similar to the proportion in humans (Nakagami et al., 2010). Given the fact that TP is involved in abscisic acid (ABA) signaling (Ghelis et al., 2008), gibberellin responses (Fu et al., 2002), cold stress (Sangwan et al., 2001), and sugar responses (Ritsema et al., 2009), TP is considered to be multiply involved in not only plant growth and development but also defense responses to biotic and abiotic stresses. 
Notably, Nemoto et al. (2015) recently reported that Arabidopsis calcium-dependent protein kinase (CPK)-related protein kinases [CRK2 (At3g19100) and CRK3 (At2g46700)] phosphorylate Tyr residues of beta-tubulin and an array of transcription factors (TFs), including ethylene response factor 13 (ERF13) (At2g44840), WRKY DNA-binding protein 14 (WRKY14) (At1g30650), ERF subfamily B-4 member ERF/AP2 transcription factor 2.6 (RAP2.6) (At1g43160), and cryptochrome-interacting basic-helix-loop-helix 5 (CIB5) (At1g26260). The transcript level of ERF13 in Arabidopsis leaves is responsive to exogenous ABA and jasmonate (JA) application, suggesting that ERF13 is relevant to plant stress responses (Lee et al., 2010; Schweizer et al., 2013). RAP2.6, another member of the ERF family, has also been shown to function in plant defense responses to nematodes, $\mathrm{ABA}$, salt and osmotic stresses (Zhu et al., 2010; Ali et al., 2013; Guo and Sun, 2017). The same holds true for the WRKY gene family, which plays key roles in plant stress responses, including toward biotic stress (Eulgem et al., 2000; Chen et al., 2012; Birkenbihl et al., 2018). Notably, in Coptis japonica, TP has been proposed to enhance the nuclear localization, DNA-binding activity and transactivation of a WRKY involved in regulating the biosynthesis of the defensive products benzylisoquinoline alkaloids (Yamada and Sato, 2016). It is therefore clear that TP plays central roles in cellular signaling of plant stress responses. CRK2 and CRK3 share $57.7 \%$ amino acid identity, and they share a serine (Ser)/threonine (Thr) kinase domain and a degenerate calcium-binding EF-hand motif. In spite of the structural similarity of CRKs to typical Ser/Thr-type protein kinases, CRK2 and CRK3 preferentially phosphorylate tyrosine residues in the absence of calcium (Nemoto et al., 2015).

On the other hand, Ser/Thr phosphorylation has been classically focused on regarding its relevance to plants' stress responses. For instance, it has been elucidated that CPK2 (NtCDPK2) modulates the activation level of stress-induced mitogen-activated protein kinases (MAPKs), leading to increased levels of the defense-associated phytohormones JA, 12-oxophytodienoic acid, and ethylene in tobacco (Ludwig et al., 2005). Moreover, Arabidopsis AtCPK3 and AtCPK13 have been reported to activate a heat shock TF (HsfB2a) involved in activation of the defense gene PDF1.2 in Arabidopsis plants infested by larvae of the generalist herbivore Spodoptera litura (Nagamangala Kanchiswamy et al., 2010). In contrast to these Ser/Thr kinases, however, the nature of Tyr kinases that act in plant defense responses remains obscure. We therefore focused on CRKs involved in the phosphorylation of defense-associated TFs. Here we show that CRK2 and CRK3 play a central role in eliciting defense responses of Arabidopsis host plants against the generalist herbivore S. litura. Moreover, since CRKs are also known to be involved in gibberellin signaling through the phosphorylation of GARU (gibberellin receptor RING E3 ubiquitin ligase), leading to ubiquitin-dependent degradation of gibberellin receptor (GID1) in Arabidopsis seedlings (Nemoto et al., 2017), phenotypic analyses were carried out using CRK overexpression and mutant lines.

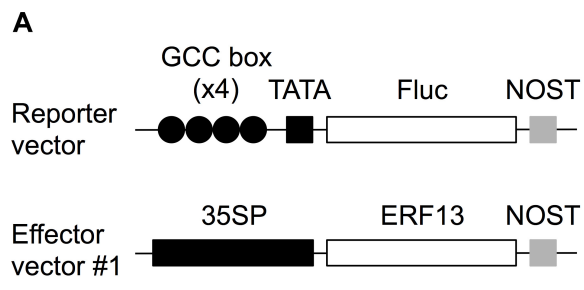

B

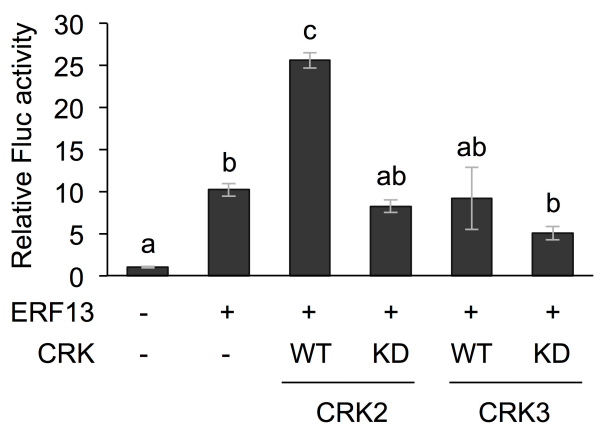

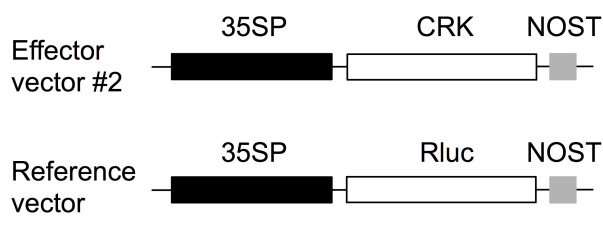

C

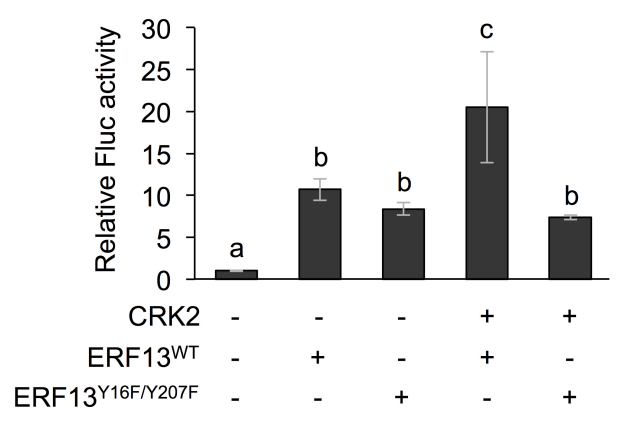

FIGURE 1 | Dual luciferase (LUC) activity mediated through CRK-activated ERF13. (A) Schematic diagram of the reporter and effector vectors used in dual LUC assays. (B) Four inverted repeats of GCC-box fused to a minimal TATA-box and a firefly LUC (Fluc) reporter gene. Transient activation of the reporter gene according to co-expression with (+) or without (-) ERF13, wild-type (WT) and kinase dead-mutant (KD) of CRK2 or CRK3 in Arabidopsis protoplasts was assessed. (C) Likewise, transient activation of the reporter gene according to co-expressed effector(s), CRK2, WT of ERF13 (ERF13WT), or ERF13 mutant deficient in CRK-phosphorylated sites (ERF13 ${ }^{\mathrm{Y} 16 \mathrm{~F} / \mathrm{Y} 207 \mathrm{~F}}$ ) in Arabidopsis protoplast cells was assessed. Data represent the mean and standard error $(n=3)$. Renilla luciferase (Rluc) activity was used to normalize for the efficiency of transformation. Means indicated by different small letters are significantly different, based on a one-way ANOVA with post hoc Tukey's HSD ( $P<0.05)$. NOST, nopaline synthase terminator; 35SP, cauliflower mosaic virus 35S promoter; and TATA, TATA-box. 


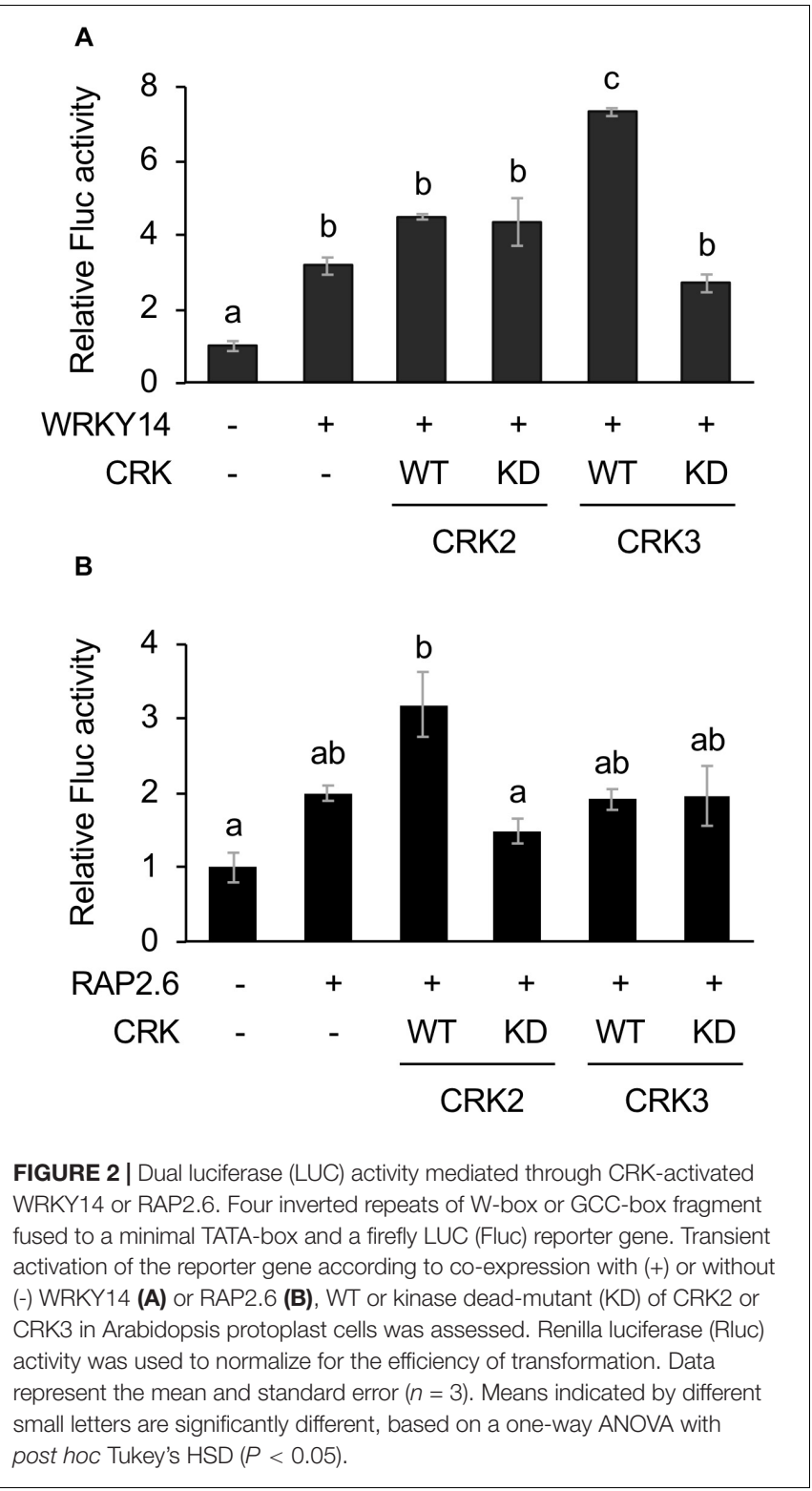

\section{MATERIALS AND METHODS}

\section{Plants}

Wild-type (WT) Arabidopsis ecotype Col-0 plants, CRK T-DNA insertion mutants [crk2 [Salk_090938C], and crk3 [Salk_128719C] (Nemoto et al., 2015)], ABA INSENSITIVE 1 mutant [abi1-1 (Allen et al., 1999)], ETHYLENE INSENSITIVE 2 mutant [ein2 (Solano et al., 1998)], and transgenic plants overexpressing CRK2 or CRK3 (see below) were grown in plastic pots for $4-5$ weeks in a growth chamber at $22 \pm 1^{\circ} \mathrm{C}$ with a photoperiod of $14 \mathrm{~h}\left(80 \mu \mathrm{E} \mathrm{m}^{-2} \mathrm{~s}^{-1}\right)$. WT of Arabidopsis ecotype Landsberg erecta (Ler) and its erf13 mutant (CS26912) were grown in these same growth conditions. The CORONATINE INSENSITIVE1 (COI1) mutant (coil-1; Col-0 background) seeds were germinated on 1/2 Murashige and Skoog (MS) medium

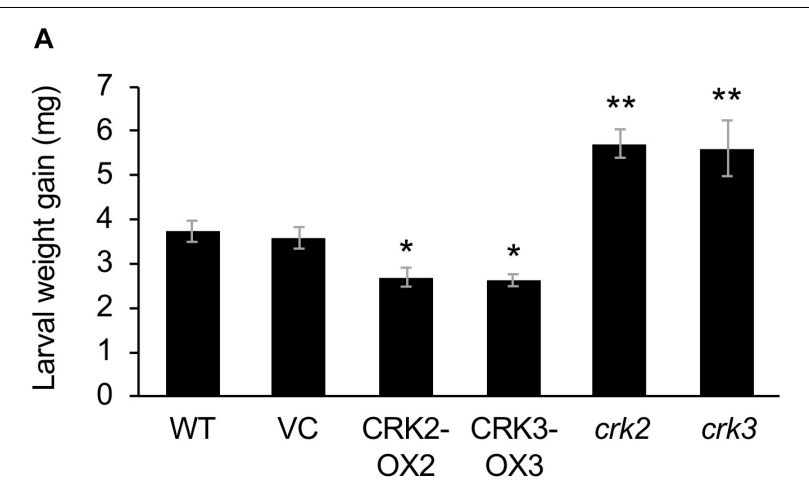

B

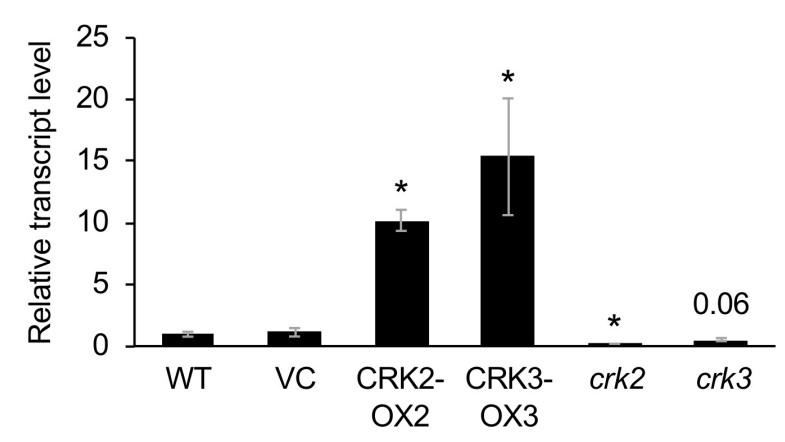

FIGURE 3 | Defense property of wild type (WT), crk mutants, and CRK-overexpressing lines. (A) The net body weight that Spodoptera litura larvae gained during 3 days after they had been placed on potted plants of WT, crk2, or crk3 mutants, CRK-overexpressing lines (CRK2-OX2 and CRK3-OX3) and their vector control (VC) lines. Data represent the mean and standard error $(n=16-22)$. (B) Transcript levels of a defensin gene PDF1.2 in the leaves of WT, crk mutants, CRK-overexpressing plants, and VC plants. Transcript levels of genes were measured by RT-qPCR and normalized by those of ACT8. Data represent the mean and standard error $(n=4$ for VC, CRK2-OX2, and CRK3-OX3; $n=7$ for WT, crk2, and crk3). Data marked with an asterisk are significantly different from those of WT (A) or VC (B), based on a one-way ANOVA with Holm's sequential Bonferroni post hoc test $\left({ }^{*} P<0.01,{ }^{*} 0.01 \leq P<0.05\right)$. Otherwise, the mean followed by $P$ value is marginally different from the control value.

supplemented with $2 \%$ sucrose, $0.8 \%$ agar, and $50 \mu \mathrm{M}$ methyl jasmonate (MeJA, Wako Pure Chemical Industrials, Ltd., Osaka, Japan) to screen the individuals showing normal root growth for 2 weeks (Xie et al., 1998). The screened plants were transferred and grown in plastic pots for an additional 3 weeks.

\section{Chemical and Herbivore Treatments}

S. litura were reared on an artificial diet (Insecta LF, Nihon Nosan Nogyo Ltd., Tokyo, Japan) in the laboratory at $24 \pm 1^{\circ} \mathrm{C}$ with a photoperiod of $16 \mathrm{~h}$. For herbivore treatment, four thirdinstar larvae per plant were released on potted Arabidopsis plants in a growth chamber for $24 \mathrm{~h}$. After chemical and herbivore treatment, all the plants were incubated at $22 \pm 1^{\circ} \mathrm{C}(14 \mathrm{~h}$ photoperiod at a light intensity of $80 \mu \mathrm{E} \mathrm{m}^{-2} \mathrm{~s}^{-1}$ ).

Arabidopsis plants were evenly sprayed with $1 \mathrm{~mL}$ of aqueous solutions $(0.1 \%(\mathrm{v} / \mathrm{v})$ ethanol) of MeJA (Wako Pure Chemical industrials; $0.2 \mathrm{mM}$ ), 1-aminocyclopropane-1-carboxylic acid (ACC, Wako Pure Chemical Industries; $0.01 \mathrm{mM}$ ) or ABA 

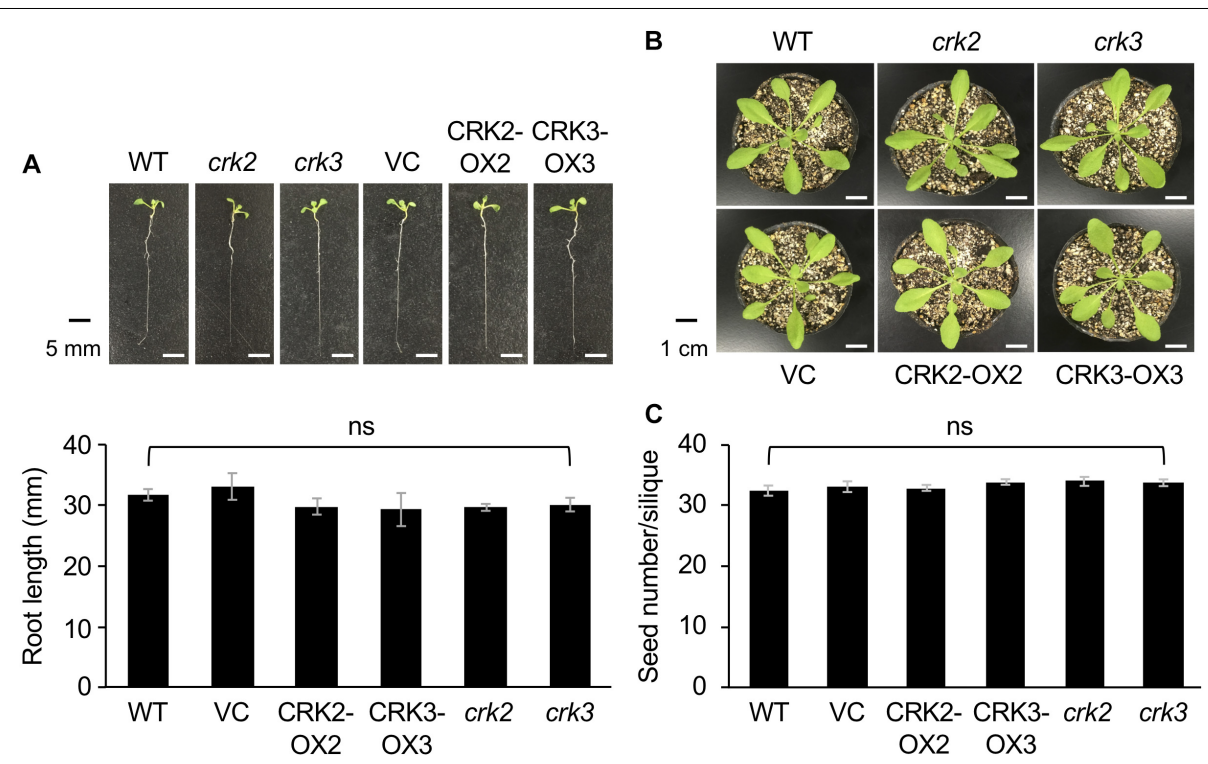

FIGURE 4 | The phenotype and root length of seedlings (A) and rosette plants (B), and the numbers of seeds (C) of wild type (WT), crk2, and crk3 mutants, CRK-overexpressing lines (CRK2-OX2 and CRK3-OX3), and their VC line. Plant seedlings were grown on medium for 14 days, and rosette plants and plants during the harvest time were grown on soil for 4 weeks and $\sim 8$ weeks, respectively. Means and standard errors of root lengths were determined using 12 individual seedlings. Means and standard errors of the numbers of seeds were determined using five pods from eight individual plants. ns, not significant based on a one-way ANOVA.

(Tokyo Chemical Industry Co., Ltd., Tokyo, Japan; $0.1 \mathrm{mM}$ ) and incubated in a growth chamber for up to $24 \mathrm{~h}$.

\section{Primers}

Primers used for all the polymerase chain reactions (PCRs) in this study are listed in Supplementary Table S1.

\section{Protoplast Preparation and Transfection}

The full-length coding region of CRK2, CRK3, or ERF13 was cloned into the p35S $\Omega-G W-N O S T$ vector [cauliflower mosaic virus $35 \mathrm{~S}$ promoter (35SP):: $\Omega$ sequence (translation enhancer)::the Gateway cassette $(\mathrm{GW})$ region::Nopaline synthase terminator (NOST) (Nemoto et al., 2015)] using the Gateway cloning system (Thermo Fisher Scientific, Waltham, MA, United States). The kinase dead (KD) form mutants of CRK2 (Lys 176 to Arg $\left[\mathrm{CRK}{ }^{\mathrm{KD}}\right]$ ) and CRK3 (Lys 175 to Arg $\left[\mathrm{CRK} 3{ }^{\mathrm{KD}}\right]$ ), and ERF13 mutants $\left(\mathrm{Tyr}_{16}\right.$ to Phe and $\mathrm{Tyr}_{207}$ to Phe [ERF13 $\left.{ }^{\mathrm{Y} 16 \mathrm{~F} / \mathrm{Y} 207 \mathrm{~F}}\right]$ ) were generated using a PrimeSTAR Mutagenesis Basal Kit (Takara Bio Inc., Otsu, Japan) according to the manufacturer's instructions (Nemoto et al., 2015). These cDNAs were also inserted into the GW region of

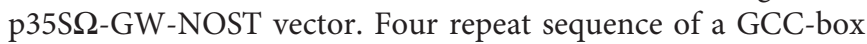
(AGCCGCC) fragment or a W-box (TTTGACC) fragment was fused to a minimal TATA box::a firefly luciferase (Fluc) reporter gene::NOST in the pMA cloning vector (Thermo Fisher Scientific, Waltham, MA, United States). The map of the representative vectors used is shown in Figure 1.

Protoplast isolation from Arabidopsis leaves was performed as previously described ( $\mathrm{Wu}$ et al., 2009). The peeled leaves (4-5-week-old plants), still adhering to the tape, were transferred to a Petri dish containing $10 \mathrm{ml}$ of enzyme solution (2\% (w/v) cellulase "Onozuka” R10 [Yakult Pharmaceutical Industry, Tokyo, Japan], 0.3\% (w/v) macerozyme "Onozuka” R10 [Yakult Pharmaceutical Industry], $0.4 \mathrm{M}$ mannitol, $10 \mathrm{mM} \mathrm{CaCl}$, and $5 \mathrm{mM}$ MES [pH 5.7]). The leaf tissues were incubated at room temperature for $1 \mathrm{~h}$ until the protoplasts were sufficiently released into the solution. The protoplasts isolated were diluted with an equal volume of W5 solution $(154 \mathrm{mM}$ $\mathrm{NaCl}, 125 \mathrm{mM} \mathrm{CaCl}_{2}, 5 \mathrm{mM} \mathrm{KCl}$, and $2 \mathrm{mM}$ MES [pH 5.7]) and filtered with filter paper to remove undigested leaf tissues. The protoplast suspension was centrifuged at $100 \mathrm{~g}$ for $2 \mathrm{~min}$ and re-suspended with W5 solution to adjust it to $2 \times 10^{5}$ cells $\mathrm{ml}^{-1}$. The protoplast suspension was centrifuged again and finally resuspended in an equal volume of modified MMG solution (0.4 M mannitol, $15 \mathrm{mM} \mathrm{MgCl}_{2}$, and 5 mM MES [pH 5.7]).

Polyethylene glycol-mediated DNA transfection was performed as previously described (Yoo et al., 2007). The protoplast suspension $(100 \mu l)$ was supplemented with a mixture of vectors carrying 35SP::CRK (CRK2 ${ }^{\mathrm{WT}}$, $\mathrm{CRK} 2^{\mathrm{KD}}, \mathrm{CRK} 3^{\mathrm{WT}}$, or CRK3 $\left.{ }^{\mathrm{KD}}\right):: \mathrm{NOST}$, 35SP::TF $\left(\mathrm{ERF} 13^{\mathrm{WT}}\right.$, ERF13 ${ }^{\mathrm{Y} 16 \mathrm{~F} / \mathrm{Y} 207 \mathrm{~F}}$, WRKY14, or RAP2.6)::NOST, GCC-box or W-box::TATA::Fluc::NOST and reference (35SP::Renilla luciferase [Rluc]::NOST) vector at a ratio of $4: 5: 5: 1$ to protoplast suspension with $110 \mu \mathrm{l}$ PEG solution [40\% (w/v) polyethylene glycerol, $0.4 \mathrm{M}$ mannitol, and $\left.0.1 \mathrm{M} \mathrm{Ca}\left(\mathrm{NO}_{3}\right)_{2} 4 \mathrm{H}_{2} \mathrm{O}\right]$. The transfection was carried out at room temperature for $5 \mathrm{~min}$ and stopped by adding $400 \mu \mathrm{l}$ of W5 solution. The protoplasts were collected by centrifugation at $100 \mathrm{~g}$ for $2 \mathrm{~min}$ and resuspended with $500 \mu \mathrm{l}$ of WI solution (5 mM MES [pH 5.7], 0.4 M mannitol, and $20 \mathrm{mM} \mathrm{KCl}$ ) and incubated in a 12-well tissue culture plate at room temperature overnight. 


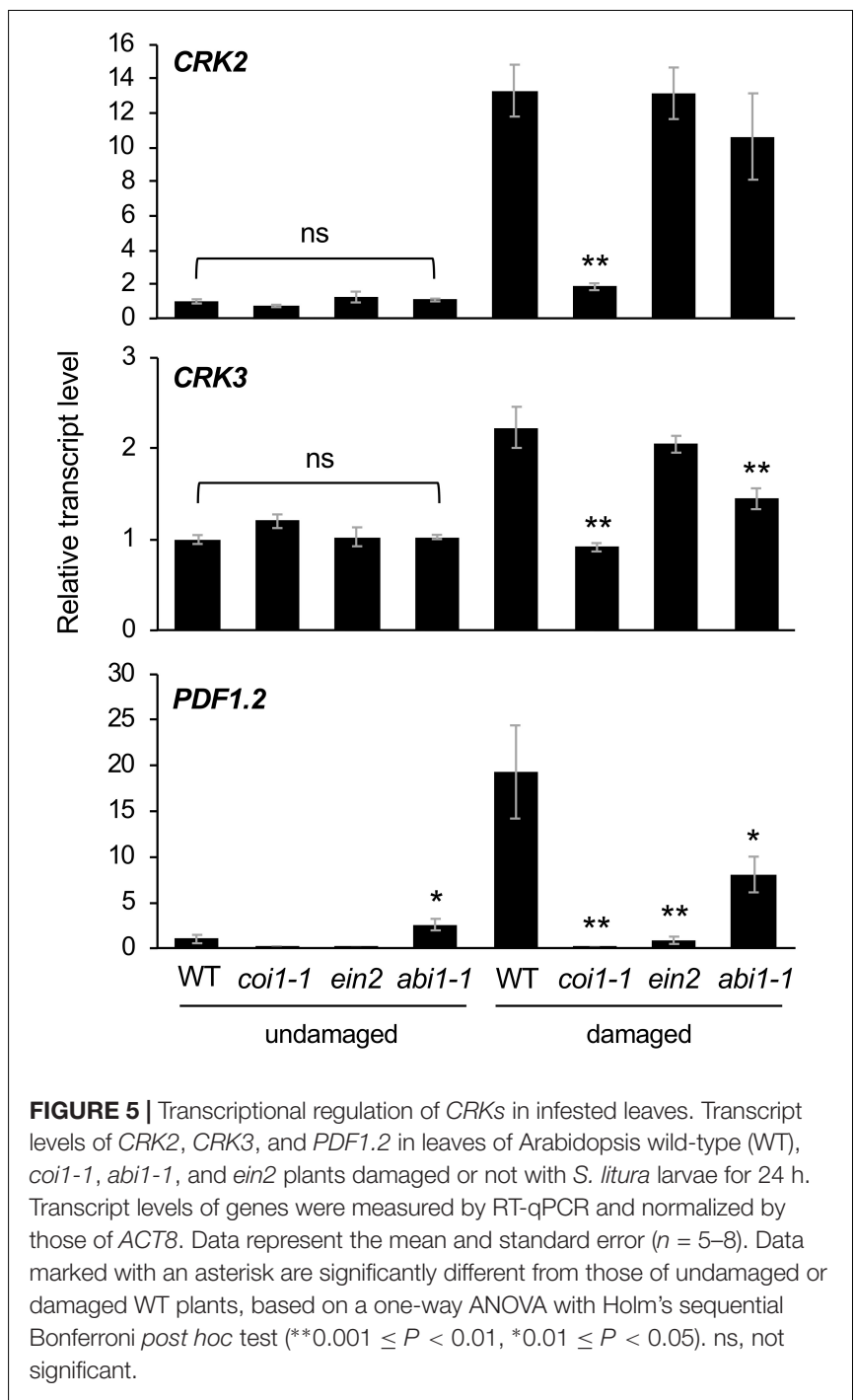

\section{Luciferase (LUC) Assay}

The LUC assay was performed as previously described (Luehrsen et al., 1992). The protoplasts were collected by centrifugation at $100 \mathrm{~g}$ for $2 \mathrm{~min}$, and re-suspended with $100 \mu \mathrm{l}$ of EX buffer (50 mM Tris- $\mathrm{HCl}[\mathrm{pH} 8.0], 150 \mathrm{mM} \mathrm{NaCl}$, and $0.5 \%(\mathrm{v} / \mathrm{v})$ Triton $\mathrm{X}-100$ ). The protoplasts were again centrifuged at $20,000 \mathrm{~g}$ for $10 \mathrm{~min}$ at $4^{\circ} \mathrm{C}$, and $10 \mu \mathrm{l}$ of supernatant was used for a LUC assay. LUC activity was measured with a 1420 Luminescence Counter ARVO Light (Perkin Elmer, Waltham, MA, United States) using the Dual-Luciferase ${ }^{\circledast}$ Reporter assay system (Promega, Madison, WI, United States). Fluc activity produced due to the transfected reporter construct was expressed as the value normalized by the Rluc activity produced due to the co-transfected reference vector. Replicate analyses were conducted with 3 independent samples.

\section{Generation of Transgenic Arabidopsis Plants}

The full-length coding region of CRK2 or CRK3 was inserted into binary vector pMDC32 (2x 35SP::GW::NOST) using the Gateway cloning system (see above). The resulting vector, pMDC32CRK2, pMDC32-CRK3 or pMDC32 [vector control (VC)], was transformed into Agrobacterium tumefaciens strain EHA105 by electroporation. WT Arabidopsis plants that had been grown for about 6-7 weeks were transformed via the floral-dip transformation method (Clough and Bent, 1998). Transgenic T1 seeds from each transformant were tested for germination on 1/2 MS medium supplemented with $30 \mathrm{mg} \mathrm{l}^{-1}$ hygromycin. T2 seeds harvested from each individual T0 plant that showed ca. 3:1 segregation ratio was tested for hygromycin-resistance again. T3 homozygous plant lines were used for further analyses.

\section{RNA Extraction, cDNA Synthesis and Quantitative PCR (qPCR)}

RNA extraction, first-strand cDNA synthesis and quantitative PCR were performed according to the method described previously (Ali et al., 2019).

\section{Herbivore Assay}

We performed assays to assess the growth of $S$. litura larvae at $22 \pm 1^{\circ} \mathrm{C}\left(14 \mathrm{~h}\right.$ photoperiod at $\left.80 \mu \mathrm{E} \mathrm{m}^{-2} \mathrm{~s}^{-1}\right)$. Third-instar larvae were initially weighed $(1.7-2.1 \mathrm{mg})$, and each larva was released onto a potted plant for 3 days. The net body weight that $S$. litura larvae gained each of the following 3 days was determined. When a larva died or was lost during the assay, we excluded that sample, and final replicate analyses were conducted with 16-22 independent samples.

\section{Root Length Measurement}

Plant seedlings (14 days old) were grown on 1/2 MS medium. Root lengths were determined using ImageJ software [version 1.50i; (Schneider et al., 2012)].

\section{Statistical Analysis}

We performed $t$-tests for pairwise analysis and one-way ANOVA with Holm's sequential Bonferroni post hoc test or Tukey's HSD test using the program ${ }^{1}$ for comparing multiple samples.

\section{RESULTS}

\section{In vivo Function of CRK2 in Transactivation of ERF13}

Both CRK2 and CRK3 phosphorylate ERF13 at two Tyr residues (Y16 and Y207) (Nemoto et al., 2015). To investigate the roles of CRK-promoted TP in ERF13 transactivation, CRKs were expressed together with ERF13 as an activator of a reporter (firefly LUC [Fluc]) gene coexpressed under the control of a chimeric promoter that consisted of four inverted repeats of GCC-box [ERF-binding cis-element (Fujimoto et al., 2000)] fused to a minimal TATA-box, in Arabidopsis mesophyll protoplasts (Figure 1A). Expression of ERF13 caused a 10-fold increase of Fluc activity in comparison to the activity in the absence of ERF13 (Figure 1B). When WT CRK2 $\left(\mathrm{CRK}^{\mathrm{WT}}\right)$ was

\footnotetext{
${ }^{1}$ http://astatsa.com/OneWay_Anova_with_TukeyHSD/
} 

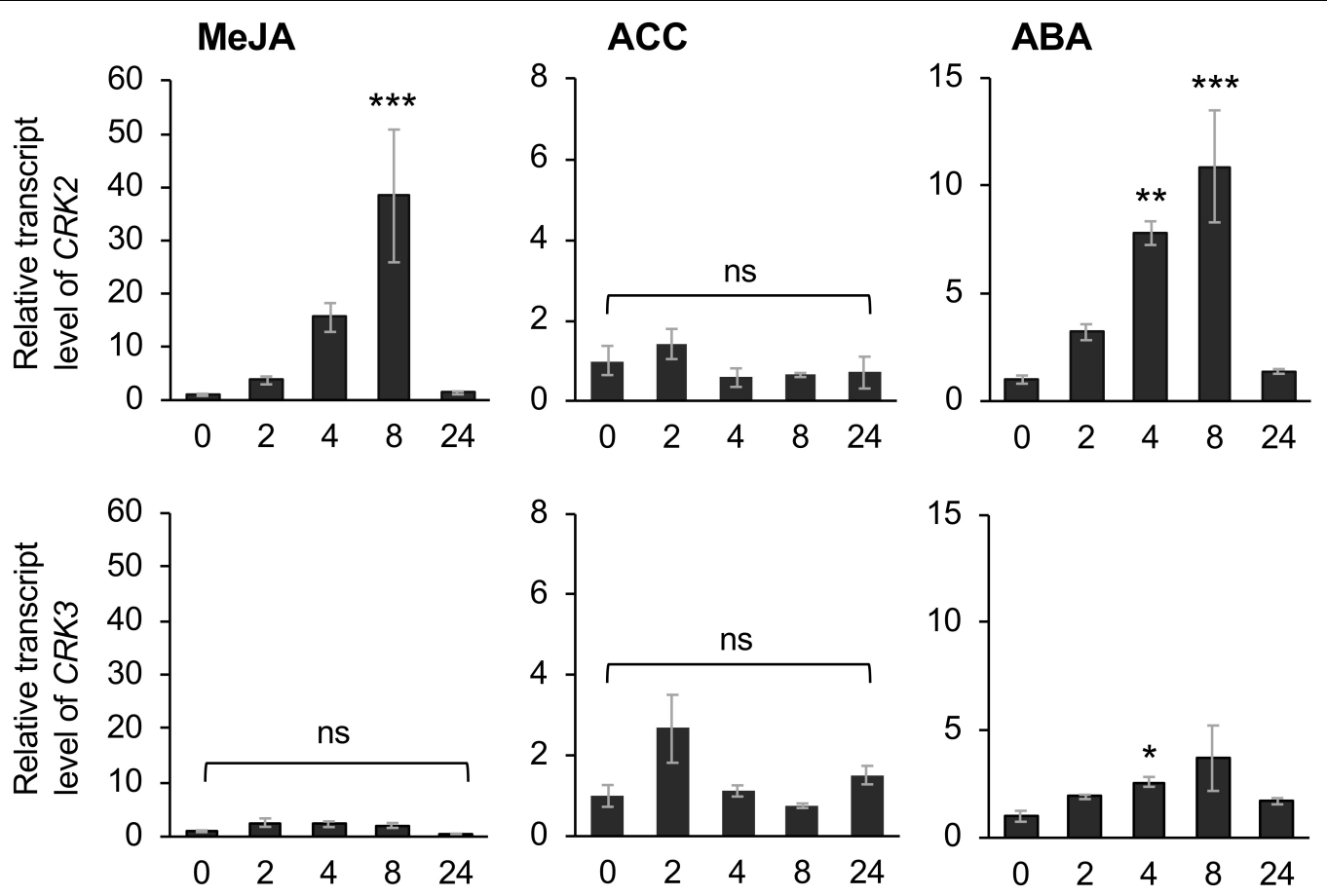

Time after treatment $(\mathrm{h})$

FIGURE 6 | Phytohormone-induced regulation of CRK expression. Transcript levels of CRK2 and CRK3 in leaves of Arabidopsis wild-type plants in response to exogenous application of methyl jasmonate (MeJA), an ethylene precursor [1-aminocyclopropane-1-carboxylic acid (ACC)], abscisic acid (ABA) for up to 24 h. Transcript levels of genes were measured by RT-qPCR and normalized by those of ACT8. Data represent the mean and standard error ( $n=6)$. Data marked with an asterisk are significantly different from those of undamaged or damaged WT plants, based on a one-way ANOVA with Holm's sequential Bonferroni post hoc test $\left({ }^{* * *} P<0.001,{ }^{* *} 0.001 \leq P<0.01 ; * 0.01 \leq P<0.05\right)$. ns, not significant.

coexpressed with ERF13, an additional 2.5-fold increase of Fluc activity was detected. However, transactivation of ERF13 was not caused by either CRK3 or kinase domain-mutant KD CRK2 (CRK2 ${ }^{\mathrm{KD}}$, whose lack of kinase activity has been shown previously; Nemoto et al., 2015, 2017) when they were concomitantly expressed in the cells. Moreover, when an ERF13 mutant deficient in CRK-phosphorylated sites $\left(\mathrm{ERF} 13^{\mathrm{Y} 16 \mathrm{~F} / \mathrm{Y} 207 \mathrm{~F}}\right)$, instead of WT ERF13 (ERF13 ${ }^{\mathrm{WT}}$ ), was co-expressed with CRK2, the Fluc activity declined to the basal level achieved by the expression of ERF13 ${ }^{\mathrm{WT}}$ alone (Figure 1C).

\section{Transactivation of WRKY14 and RAP2.6 by CRK}

CRK2 and CRK3 are also able to phosphorylate WRKY14 (Nemoto et al., 2015), one of the WRKY members involved in an array of plant defense responses (Skibbe et al., 2008; Bakshi and Oelmuller, 2014; Li et al., 2015). Moreover, RAP2.6, another $\mathrm{AP} 2 / \mathrm{ERF}$ protein member involved in plant stress responses (Krishnaswamy et al., 2011; Ali et al., 2013), has been shown to be phosphorylated by CRK2 but not CRK3 (Nemoto et al., 2015). We therefore explored those two TFs as substrate targets for CRKs, utilizing the transient Fluc expression system in protoplast cells, using the two cis-elements, i.e., a W-box and a GCC-box for WRKY14 (Chen et al., 2012) and RAP2.6 (Zhu et al., 2010), respectively.

The Fluc activity was increased by WRKY14 expression. This activity marginally tended to be elevated by coexpression of WT CRK3 $\left(\mathrm{CRK} 3^{\mathrm{WT}}\right.$ ) (Figure 2A). However, this transactivation was achieved by neither $\mathrm{CRK} 3^{\mathrm{KD}}$ nor CRK2 (CRK2 ${ }^{\mathrm{WT}}$ or $\left.\mathrm{CRK} 2{ }^{\mathrm{KD}}\right)$. In contrast, Fluc activity, which was only marginally increased by expression of RAP2.6, was elevated by coexpression of CRK2 ${ }^{\mathrm{WT}}$ (Figure 2B). Again, this transactivation was not achieved by coexpression of $\mathrm{CRK} 2^{\mathrm{KD}}$ or $\mathrm{CRK} 3\left(\mathrm{CRK} 3^{\mathrm{WT}}\right.$ or $\left.\mathrm{CRK} 3^{\mathrm{KD}}\right)$.

\section{Defense Ability and Growth/Development of CRK Mutants and Overexpressing Lines}

We obtained two lines and three lines of CRK2- or CRK3-overexpressing plants, respectively. Two respective representative lines (CRK2-OX2 and CRK3-OX3) exhibited 170-fold and 70-fold increased levels of CRK2 and CRK3 expression under the constitutive 35SP, respectively, compared to the levels in leaves of the VC line (Supplementary Figure S1).

These transgenic lines exhibited lower development of larvae of the generalist herbivore $S$. litura hosted on the potted plants for 3 days, compared to that on VC plants (Figure 3A). This was in agreement with the constitutively elevated expression levels 
of the JA-inducible plant defensin gene PDF1.2 (Manners et al., 1998) in leaves of these two transgenic lines (Figure 3B). In contrast, crk2 and crk3 knockdown mutants (Nemoto et al., 2015) exhibited enhanced development of larvae on the potted plants during 3 days, in accord with the constitutively lower expression level of PDF1.2 in their leaves (Figures 3A,B). The GCC-box located at -255 to -261 in the PDF1.2 promoter has been shown to play a key role in conferring JA responsiveness to PDF1.2 expression (Brown et al., 2003). Putative W-boxes (TGACC/T) are also located at -388 to $-384,-773$ to -768 , and -828 to -823 in the PDF1.2 promoter upstream region.

None of the transgenic lines or mutants of CRKs showed any marked differences in plant growth, development or morphology, including root growth, vegetative stage development, or seed number (Figure 4).

\section{Transcriptional Regulation of CRK2 and CRK3 via Phytohormone Signaling for Defense Responses}

Finally, Arabidopsis mutant plants defective in JA signaling (coi1-1) (Xie et al., 1998), ethylene signaling (ein2) (Ju and Chang, 2015), and ABA signaling (abi1-1) (Pei et al., 1997) were assessed to evaluate the involvement of hormone signaling in $C R K$ activation during damage by $S$. litura (Figure 5). In comparison to the induction of transcripts of CRK2 and CRK3 in WT leaves, coil-1 plants exhibited defective elevation of these transcript levels in leaves upon herbivory, as did PDF1.2 plants. ein2 and abi1-1 plants did not show defective elevation of transcript levels of CRK2 upon herbivory. However, abi1-1 plants showed defective elevation of the CRK3 transcript level upon herbivory. Although abi1-1 leaves showed slightly higher expression of PDF1.2 compared to WT in undamaged leaves, this issue was not further explored because it was outside the focus of the present study.

The application of exogenous phytohormone solutions to WT plants resulted in induced expression of CRK2 in leaves treated with the methyl form of JA (MeJA) or ABA but not an ethylene precursor (ACC) (Figure 6). Moreover, expression of CRK3 was elicited at $4 \mathrm{~h}$ in leaves treated with $\mathrm{ABA}$ but not MeJA or ACC for up to $24 \mathrm{~h}$.

\section{DISCUSSION}

Tyr phosphorylation mediated by Arabidopsis CRKs appeared to modulate the activities of TFs including ERF13, WRKY14, and RAP2.6 (Figures 1, 2). Although ERF13 and WRKY14 were previously shown to be phosphorylated by both CRK2 and CRK3 using an in vitro phosphorylation system (Nemoto et al., 2015), the transactivation of ERF13 and WRKY14 was achieved by CRK2 and CRK3 alone, respectively (Figures 1, 2). However, these findings are not surprising because in vitro phosphorylation activity does not always accord with the in vivo functions (Delom and Chevet, 2006). This may be because the concentrated kinase protein and/or possibly contaminating kinases from the eukaryotic protein synthesis system cause non-specific phosphorylation of substrate targets in in vitro assays. Moreover, it is known that phosphorylation modification of TFs can be responsible not only for their transactivation but also for their nuclear translocation (Liu et al., 2017) as well as enhancement of their binding to the particular cis-element of the respective promoter region (Gao et al., 2013).

According to our phenotypic characterization of loss and gain of CRK functions, it appeared that both CRK2 and CRK3 are involved in plant defense responses to $S$. litura damage (Figure 3). However, it is important to note that the erf13 mutant did not fully modulate the herbivore performance or the PDF1.2 transcript in leaves, compared to those in WT (Supplementary Figure S2), in accord with previous findings (Schweizer et al., 2013). All these facts lead us to propose a model in which multiple CRK substrates, including not only ERF13 but also WRKY14, RAP2.6 and unknown TF substrates, may individually and/or synergistically coordinate the upregulation of defense genes such as PDF1.2 (Figure 7). In addition, CRKs may control various regulatory molecules besides TFs in cellular signaling. For example, CRK2 phosphorylates GARU, a protein involved in ubiquitin-dependent degradation of the gibberellin receptor GID1 in gibberellin signaling of Arabidopsis seedlings (Nemoto et al., 2017). Both garu mutant and CRK2-OX plants enhance GID1 stabilization and DELLA degradation, indicating that CRK2 is positively involved in gibberellin signaling through the

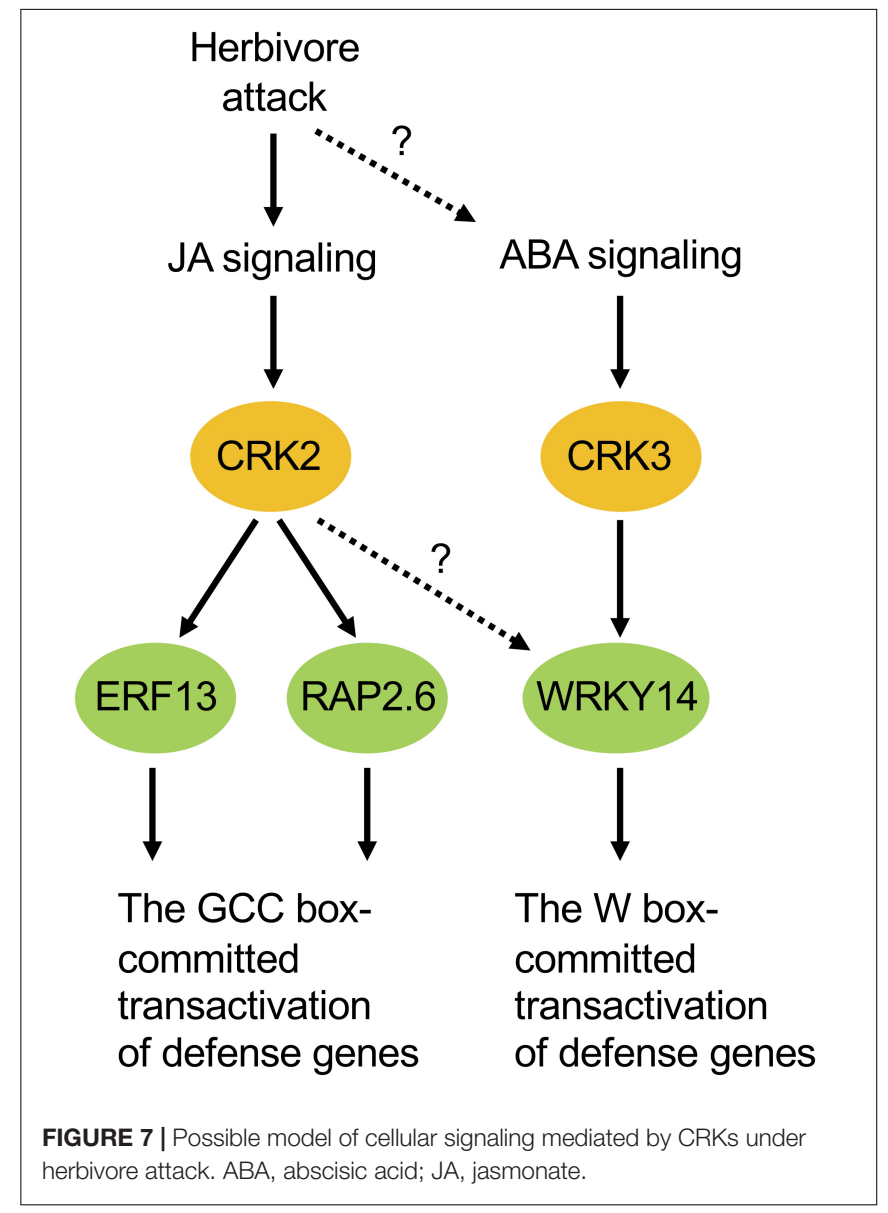


CRK2-mediated Tyr phosphorylation of GARU in Arabidopsis seedlings (Nemoto et al., 2017).

Moreover, ABA-responsive CRK3 (Figure 5) may be involved in in planta responses to not only herbivory but also pathogenesis and multiple environmental stresses. For example, WRKY, a substrate of CRK3, should especially function in gene regulation for environmental stress tolerance (Chen et al., 2012), and TP has been shown to be involved in ABA signaling (Ghelis et al., 2008), as described above. On the other hand, given the transcriptional profile of CRK2 in the leaves of MeJA-treated WT plants and infested coil-1 plants, there is no doubt that JA is a master switch for CRK2 activation in the infested leaves (Figures 5, 6, 7). In contrast to this, although ABA application activates CRK2 expression, ABA is not likely to contribute to herbivory-response signaling, considering the data observed using abi1-1. In contrast, CRK3 expression was not responsive to JA, but JA is likely involved in herbivory-response signaling according to data observed using coil-1. Regarding this, we presume that CRK3 is not directly activated by JA signaling, but probably concomitant effects from other signaling pathways such as $\mathrm{ABA}$ signaling, in concert with defense-signaling cross-talk (Erb et al., 2012), might affect CRK3 expression in coil-1 leaves during herbivory.

Finally, it should be remarked that neither CRK-OX nor mutant lines show any phenotypic defects in plant growth, development or morphology in comparison to WT plants (Figure 4). Thus, CRKs are not likely to be relevant to plant development in the normal growth condition, although this seems to be paradoxical to the above-described possible involvement of CRK2 in gibberellin signaling. We therefore propose a possible model that CRK2 does not play a significant role in the GID1/GARU system under the normal condition, in which a low threshold level of endogenous gibberellin is maintained. However, when plants suffer from threats such as a lack of nutrients or biotic/abiotic stresses, plants switch to reduced endogenous gibberellin levels (Wild et al., 2012;

\section{REFERENCES}

Ali, M. A., Abbas, A., Kreil, D. P., and Bohlmann, H. (2013). Overexpression of the transcription factor RAP2.6 leads to enhanced callose deposition in syncytia and enhanced resistance against the beet cyst nematode Heterodera schachtii in Arabidopsis roots. BMC Plant Biol. 13:47. doi: 10.1186/1471-2229-13-47

Ali, M. R. M., Uemura, T., Ramadan, A., Adachi, K., Nemoto, K., Nozawa, A., et al. (2019). The ring-type E3 ubiquitin ligase JUL1 targets the VQ-motif protein JAV1 to coordinate jasmonate signaling. Plant Physiol. 179, 1273-1284. doi: $10.1104 /$ pp.18.00715

Allen, G. J., Kuchitsu, K., Chu, S. P., Murata, Y., and Schroeder, J. I. (1999). Arabidopsis abi1-1 and abi2-1 phosphatase mutations reduce abscisic acidinduced cytoplasmic calcium rises in guard cells. Plant Cell 11, 1785-1798. doi: $10.1105 /$ tpc.11.9.1785

Bakshi, M., and Oelmuller, R. (2014). WRKY transcription factors: jack of many trades in plants. Plant Signal. Behav. 9:e27700. doi: 10.4161/psb.27700

Birkenbihl, R. P., Kracher, B., Ross, A., Kramer, K., Finkemeier, I., and Somssich, I. E. (2018). Principles and characteristics of the Arabidopsis WRKY regulatory network during early MAMP-triggered immunity. Plant J. 96, 487-502. doi: $10.1111 /$ tpj.14043

Blume-Jensen, P., and Hunter, T. (2001). Oncogenic kinase signalling. Nature 411, 355-365. doi: 10.1038/35077225
Colebrook et al., 2014), and then CRK2 is recruited to play a primary role in gibberellin signaling. In other words, CRKs do serve under certain conditions for plants' defense responses to environmental threats and plant growth/development, mediated through the assistance of phytohormone signaling.

\section{DATA AVAILABILITY}

The datasets for this manuscript are not publicly available because data has not yet been linked to any public still domains. Requests to access the datasets should be directed to G-iA, garimura@rs.tus.ac.jp.

\section{AUTHOR CONTRIBUTIONS}

TM, TU, KN, MD, TS, and G-iA contributed to the conception and design the study. TM, TU, KN, MD, and AN performed the experiments. G-iA wrote the first draft of the manuscript. TM, $\mathrm{TU}, \mathrm{KN}$, and G-iA wrote sections of the manuscript.

\section{FUNDING}

This work was financially supported in part by the JSPS KAKENHI to G-iA (16K07407) and MEXT Grants-in-Aid for Scientific Research on Innovative Areas to G-iA (18H04630 and 18H04786), and KN (18H04634).

\section{SUPPLEMENTARY MATERIAL}

The Supplementary Material for this article can be found online at: https://www.frontiersin.org/articles/10.3389/fpls.2019.00776/ full\#supplementary-material

Brown, R. L., Kazan, K., Mcgrath, K. C., Maclean, D. J., and Manners, J. M. (2003). A role for the GCC-box in jasmonate-mediated activation of the PDF1.2 gene of Arabidopsis. Plant Physiol. 132, 1020-1032. doi: 10.1104/pp.102.017814

Chen, L., Song, Y., Li, S., Zhang, L., Zou, C., and Yu, D. (2012). The role of WRKY transcription factors in plant abiotic stresses. Biochim. Biophys. Acta 1819, 120-128. doi: 10.1016/j.bbagrm.2011.09.002

Clough, S. J., and Bent, A. F. (1998). Floral dip: a simplified method for Agrobacterium-mediated transformation of Arabidopsis thaliana. Plant J. 16, 735-743. doi: 10.1046/j.1365-313x.1998.00343.x

Colebrook, E. H., Thomas, S. G., Phillips, A. L., and Hedden, P. (2014). The role of gibberellin signalling in plant responses to abiotic stress. J. Exp. Biol. 217, 67-75. doi: $10.1242 /$ jeb. 089938

Delom, F., and Chevet, E. (2006). Phosphoprotein analysis: from proteins to proteomes. Proteome Sci. 4:15.

Erb, M., Meldau, S., and Howe, G. A. (2012). Role of phytohormones in insectspecific plant reactions. Trends Plant Sci. 17, 250-259. doi: 10.1016/j.tplants. 2012.01.003

Eulgem, T., Rushton, P. J., Robatzek, S., and Somssich, I. E. (2000). The WRKY superfamily of plant transcription factors. Trends Plant Sci. 5, 199-206. doi: 10.1016/s1360-1385(00)01600-9

Fu, X., Richards, D. E., Ait-Ali, T., Hynes, L. W., Ougham, H., Peng, J., et al. (2002). Gibberellin-mediated proteasome-dependent degradation of the barley 
DELLA protein SLN1 repressor. Plant Cell 14, 3191-3200. doi: 10.1105/tpc. 006197

Fujimoto, S. Y., Ohta, M., Usui, A., Shinshi, H., and Ohme-Takagi, M. (2000). Arabidopsis ethylene-responsive element binding factors act as transcriptional activators or repressors of GCC box-mediated gene expression. Plant Cell 12, 393-404. doi: 10.1105/tpc.12.3.393

Gao, X., Chen, X., Lin, W., Chen, S., Lu, D., Niu, Y., et al. (2013). Bifurcation of Arabidopsis NLR immune signaling via $\mathrm{Ca}^{2+}$-dependent protein kinases. PLoS Pathog. 9:e1003127. doi: 10.1371/journal.ppat.1003127

Ghelis, T., Bolbach, G., Clodic, G., Habricot, Y., Miginiac, E., Sotta, B., et al. (2008). Protein tyrosine kinases and protein tyrosine phosphatases are involved in abscisic acid-dependent processes in Arabidopsis seeds and suspension cells. Plant Physiol. 148, 1668-1680. doi: 10.1104/pp.108.124594

Guo, R., and Sun, W. (2017). Sumoylation stabilizes RACK1B and enhance its interaction with RAP2.6 in the abscisic acid response. Sci. Rep. 7:44090. doi: $10.1038 /$ srep44090

Ju, C., and Chang, C. (2015). Mechanistic insights in ethylene perception and signal transduction. Plant Physiol. 169, 85-95. doi: 10.1104/pp.15.00845

Krishnaswamy, S., Verma, S., Rahman, M. H., and Kav, N. N. (2011). Functional characterization of four APETALA2-family genes (RAP2.6, RAP2.6L, DREB19 and DREB26) in Arabidopsis. Plant Mol. Biol. 75, 107-127. doi: 10.1007/s11103010-9711-7

Lee, S. J., Park, J. H., Lee, M. H., Yu, J. H., and Kim, S. Y. (2010). Isolation and functional characterization of CE1 binding proteins. BMC Plant Biol. 10:277. doi: 10.1186/1471-2229-10-277

Li, R., Zhang, J., Li, J., Zhou, G., Wang, Q., Bian, W., et al. (2015). Prioritizing plant defence over growth through WRKY regulation facilitates infestation by non-target herbivores. eLife 4:e04805. doi: 10.7554/eLife.04805

Liu, K. H., Niu, Y., Konishi, M., Wu, Y., Du, H., Sun Chung, H., et al. (2017). Discovery of nitrate-CPK-NLP signalling in central nutrient-growth networks. Nature 545, 311-316. doi: 10.1038/nature22077

Ludwig, A. A., Saitoh, H., Felix, G., Freymark, G., Miersch, O., Wasternack, C., et al. (2005). Ethylene-mediated cross-talk between calcium-dependent protein kinase and MAPK signaling controls stress responses in plants. Proc. Natl. Acad. Sci. U.S.A. 102, 10736-10741. doi: 10.1073/pnas.0502954102

Luehrsen, K. R., Dewet, J. R., and Walbot, V. (1992). Transient expression analysis in plants using firefly luciferase reporter gene. Methods Enzymol. 216, 397-414. doi: 10.1016/0076-6879(92)16037-k

Manners, J. M., Penninckx, I. A. M. A., Vermaere, K., Kazan, K., Brown, R. L., Morgan, A., et al. (1998). The promoter of the plant defensin gene PDF1.2 from Arabidopsis is systemically activated by fungal pathogens and responds to methyl jasmonate but not to salicylic acid. Plant Mol. Biol. 38, 1071-1080.

Nagamangala Kanchiswamy, C., Takahashi, H., Quadro, S., Maffei, M. E., Bossi, S., Bertea, C., et al. (2010). Regulation of Arabidopsis defense responses against Spodoptera littoralis by CPK-mediated calcium signaling. BMC Plant Biol. 10:97. doi: 10.1186/1471-2229-10-97

Nakagami, H., Sugiyama, N., Mochida, K., Daudi, A., Yoshida, Y., Toyoda, T., et al. (2010). Large-scale comparative phosphoproteomics identifies conserved phosphorylation sites in plants. Plant Physiol. 153, 1161-1174. doi: 10.1104/pp. 110.157347

Nemoto, K., Ramadan, A., Arimura, G., Imai, K., Tomii, K., Shinozaki, K., et al. (2017). Tyrosine phosphorylation of the GARU E3 ubiquitin ligase promotes gibberellin signalling by preventing GID1 degradation. Nat. Commun. 8:1004. doi: 10.1038/s41467-017-01005-5

Nemoto, K., Takemori, N., Seki, M., Shinozaki, K., and Sawasaki, T. (2015). Members of the plant CRK superfamily are capable of trans- and autophosphorylation of tyrosine residues. J. Biol. Chem. 290, 16665-16677. doi: 10.1074/jbc.M114.617274

Pei, Z. M., Kuchitsu, K., Ward, J. M., Schwarz, M., and Schroeder, J. I. (1997). Differential abscisic acid regulation of guard cell slow anion channels in Arabidopsis wild-type and abi1 and abi2 mutants. Plant Cell 9, 409-423. doi: 10.1105/tpc.9.3.409

Ritsema, T., Brodmann, D., Diks, S. H., Bos, C. L., Nagaraj, V., Pieterse, C. M. J., et al. (2009). Are small GTPases signal hubs in sugar-mediated induction of fructan biosynthesis? PLoS One 4:e6605. doi: 10.1371/journal.pone.0006605

Sangwan, V., Foulds, I., Singh, J., and Dhindsa, R. S. (2001). Cold-activation of Brassica napus BN115 promoter is mediated by structural changes in membranes and cytoskeleton, and requires $\mathrm{Ca}^{2+}$ influx. Plant J. 27, 1-12. doi: 10.1046/j.1365-313x.2001.01052.x

Schneider, C. A., Rasband, W. S., and Eliceiri, K. W. (2012). NIH Image to ImageJ: 25 years of image analysis. Nat. Methods 9, 671-675. doi: 10.1038/nmeth.2089

Schweizer, F., Bodenhausen, N., Lassueur, S., Masclaux, F. G., and Reymond, P. (2013). Differential contribution of transcription factors to Arabidopsis thaliana defense against Spodoptera littoralis. Front. Plant Sci. 4:13. doi: 10.3389/fpls. 2013.00013

Skibbe, M., Qu, N., Galis, I., and Baldwin, I. T. (2008). Induced plant defenses in the natural environment: Nicotiana attenuata WRKY3 and WRKY6 coordinate responses to herbivory. Plant Cell 20, 1984-2000. doi: 10.1105/tpc.108.058594

Solano, R., Stepanova, A., Chao, Q., and Ecker, J. R. (1998). Nuclear events in ethylene signaling: a transcriptional cascade mediated by ETHYLENEINSENSITIVE3 AND ETHYLENE-RESPONSE-FACTOR1. Genes Dev. 12, 3703-3714. doi: 10.1101/gad.12.23.3703

Wild, M., Davière, J. M., Cheminant, S., Regnault, T., Baumberger, N., Heintz, D., et al. (2012). The Arabidopsis DELLA RGA-LIKE3 is a direct target of MYC2 and modulates jasmonate signaling responses. Plant Cell 24, 3307-3319. doi: $10.1105 /$ tpc. 112.101428

Wu, F. H., Shen, S. C., Lee, L. Y., Lee, S. H., Chan, M. T., and Lin, C. S. (2009). TapeArabidopsis Sandwich - a simpler Arabidopsis protoplast isolation method. Plant Methods 5:16. doi: 10.1186/1746-4811-5-16

Xie, D. X., Feys, B. F., James, S., Nieto-Rostro, M., and Turner, J. G. (1998). COI1: an Arabidopsis gene required for jasmonate-regulated defense and fertility. Science 280, 1091-1094. doi: 10.1126/science.280.5366.1091

Yamada, Y., and Sato, F. (2016). Tyrosine phosphorylation and protein degradation control the transcriptional activity of WRKY involved in benzylisoquinoline alkaloid biosynthesis. Sci. Rep. 6:31988. doi: 10.1038/srep31988

Yoo, S. D., Cho, Y. H., and Sheen, J. (2007). Arabidopsis mesophyll protoplasts: a versatile cell system for transient gene expression analysis. Nat. Protoc. 2, 1565-1572. doi: 10.1038/nprot.2007.199

Zhu, Q., Zhang, J., Gao, X., Tong, J., Xiao, L., Li, W., et al. (2010). The Arabidopsis AP2/ERF transcription factor RAP2.6 participates in ABA, salt and osmotic stress responses. Gene 457, 1-12. doi: 10.1016/j.gene.2010.02.011

Conflict of Interest Statement: The authors declare that the research was conducted in the absence of any commercial or financial relationships that could be construed as a potential conflict of interest.

Copyright (C) 2019 Miyamoto, Uemura, Nemoto, Daito, Nozawa, Sawasaki and Arimura. This is an open-access article distributed under the terms of the Creative Commons Attribution License (CC BY). The use, distribution or reproduction in other forums is permitted, provided the original author(s) and the copyright owner(s) are credited and that the original publication in this journal is cited, in accordance with accepted academic practice. No use, distribution or reproduction is permitted which does not comply with these terms. 